\title{
Mapping of globus pallidus and ventral pallidum lesions that produce hyperkinetic treading
}

\author{
Howard C. Cromwell, Kent C. Berridge * \\ Department of Psychology, The University of Michigan, 1103 E. Huron Street, Ann Arbor, MI 48104, USA
}

Accepted 20 September 1994

\begin{abstract}
The purpose of this study was to identify sites where striatopallidal lesions produce two distinct sensory-triggered hyperkinetic syndromes: (1) exaggerated forelimb treading alone to oral taste infusions and (2) sensorimotor exaggerated treading plus enhanced aversive reactions to taste infusions. The behavioral characteristics of these syndromes have been described previously (Berridge, K.C. and Cromwell, H.C., Behav. Neurosci., 104 (1990) 778-795). Bilateral excitotoxin lesions were made using quinolinic acid $(10 \mu \mathrm{g}$ in $1 \mu \mathrm{l})$ in the caudate/putamen, nucleus accumbens, globus pallidus or ventral pallidum/substantia innominata. In order to identify the precise center, borders, severity and size of lesion sites that caused these hyperkinetic treading syndromes, neuron counts (modified fractionator technique) and glial fibrillary acidic protein immunoreactivity (GFAP-IR) densitometry were used in a stereological mapping analysis. The site of lesions that produced the hyperkinetic treading syndrome without enhanced aversion was found to be restricted to the globus pallidus (GP). Damage exceeding $60 \%$ neuron loss bilaterally within a $0.8 \times 1.0 \times 1.0 \mathrm{~mm}$ subregion of the ventromedial GP produced this syndrome. The site of lesions that produced the combined syndrome of hyperkinetic treading and aversive enhancement was ventral to the globus pallidus, within the ventral pallidum/substantia innominata (VP/SI). Damage exceeding $70 \%$ neuron loss bilaterally within a $1.0 \times 0.5 \times$ $1.0 \mathrm{~mm}$ diameter subregion of the ventromedial ventral pallidum/substantia innominata produced this syndrome. This subterritory was located immediately lateral to the border of the lateral hypothalamus. Bilateral lesions to the caudate/putamen or nucleus accumbens did not produce either hyperkinetic treading syndrome. These results are discussed in terms of the connectivity of the ventral pallidal/substantia innominata and globus pallidus regions and in terms of neuropathological models of hyperkinetic disorders.
\end{abstract}

Keywords: Striatopallidal; Globus pallidus; Ventral pallidum; Hyperkinesia; Quinolinic acid; Lesion mapping

\section{Introduction}

Hyperkinetic movements of Huntington's disease [73], Gilles de la Tourette's syndrome, and tardive dyskinesia are thought to originate from disturbances in neuronal activity of the striatopallidal system $[1,6,25,68,75]$. Pathological movements of these disorders can range from brief, simple motor tics (defined as "stereotyped jerks of isolated muscles of the upper parts of the body"; see Thompson et al. [71] p. 214) to choreiform sequences (defined as "brisk, graceful series of successive, involuntary movements of consider-

\footnotetext{
* Corresponding author. Neuroscience Laboratory Building, The University of Michigan, 1103 E. Huron Street, Ann Arbor, MI 48104, USA. Fax: (1) (313) 936-2690.
}

able complexity which resemble fragments of purposeful voluntary movements"; see Carpenter [17] p. 253). Examples of the former include an abrupt shrug of the shoulder or rapid forearm extension while the latter could involve a combination of forelimb reaches intertwined with finger flexion or hand grasping motions.

Most animal models of hyperkinesia only partially replicate the characteristics of any of these disorders. In humans, hyperkinetic movements are often spontaneous after damage to particular brain regions, yet in many animal models hyperkinetic movements appear only after the administration of drugs. For example, Crossman and colleagues have produced hyperkinetic movements in the monkey by injecting bicuculline, a GABA receptor antagonist, into the lateral globus pallidus $[20,42]$. Movements analogous to chorea are seen in monkeys given levodopa or apomorphine after stri- 
atal lesions $[37,44]$. In the rat, chronic systemic injections of 3,3-iminodipropionitrile, a neurotoxin, have produced choreiform and athetoid head and neck movements [67], and intracaudate injections of a GABA antagonist combined with sensorimotor cortex damage have produced rhythmic jerking of the limbs [70]. In a few studies of non-human primates, spontaneous hyperkinesia without drug administration has been produced. Denny-Brown reported a 'wing-beating' motion in monkeys with putamen lesions [22], and Carpenter has reported hyperkinetic movements following bilateral pallidal damage in the macaque monkey [16]

Recently, a novel hyperkinetic movement pattern has been documented in the rat, which occurs after striatopallidal lesions [11]. This hyperkinetic movement pattern is perhaps the first to be reported for rodents that occurs without need of pharmacological manipulation. The movement pattern resembles both the hyperkinetic movements of Huntington's disease and of Tourette's syndrome in several respects [10,11]. In the rat, the hyperkinetic movement is triggered by oral sensory input not unlike the sensory-triggered motor tics observed in certain cases of Tourette's syndrome $[14,46]$. The hyperkinetic movement in rats is an exaggerated paw treading: a rapid, vigorous and rhythmic forelimb extension followed by retraction. Hyperkinetic treading is an exaggeration of normal but infrequent paw treading seen in intact rats in response to oral infusions of highly bitter-tasting solutions. Normal paw-treading described by Grill and Norgren [26] is composed of smaller forelimb movements ranging in amplitude from $1-5 \mathrm{~mm}$ and lasting as a series of movements typically $1-5 \mathrm{~s}$.

The pathological exaggeration of treading produced by striatopallidal lesions is manifest by an increase in incidence, amplitude, and duration of the movement pattern compared to normal rats. After large excitotoxin lesions of the posterior corpus striatum and pallidal region, $100 \%$ of rats show exaggerated treading to oral infusions of quinine (as opposed to only $10 \%$ of normal rats that show ordinary treading). The amplitude of the movements is increased 5 to 10 times (ranging from $1 \mathrm{~cm}$ to $3 \mathrm{~cm}$ in each cycle for the hyperkinetic form) and the duration of a bout is increased 3 to 10 times (ranging from 12 to $60 \mathrm{~s}$ of a 1 $\min$ trial) $[10,11]$.

A peculiar feature of some hyperkinetic disorders in humans is that the emotional state of the patient may affect the expression of the hyperkinetic movement produced by the disease [15]. For example, choreic movements become more frequent and greater in amplitude upon increased arousal or stress [45]. When a psychological 'load' is added to a motor task for patients with choreiform disorder, the choreic movements become more pronounced compared to the choreic movements seen in the same motor task ordinarily [57].
Hyperkinetic treading by the rat with a large striatopallidal lesion is similar in that it also is regulated by emotional state. In particular it is enhanced by certain aversive states. For example, some rats with large lesions of the striatopallidal system display the hyperkinetic treading to only bitter 'aversive' tasting solutions and not sweet 'palatable' tasting solutions. These rats can be converted to show the hyperkinetic treading to sweet 'palatable' tastes by associatively pairing these sweet solutions (sucrose) with $\mathrm{LiCl}$-induced illness [11]. This result suggests that motivational aversion and sensorimotor pathology interact to control the final expression of pathological treading by rats after striatopallidal damage $[11,19]$.

Hyperkinetic treading in the rat can occur in two distinct forms or syndromes after striatopallidal lesions depending apparently on properties of the lesion such as location, size or severity of neuron loss [11]. In the first syndrome (treading only or ' $\mathrm{T}$ syndrome'), rats emit hyperkinetic treading only to bitter or other unpalatable tastes, and are essentially normal in locomotion and feeding behavior. In the second syndrome (enhanced aversion plus treading or ' $\mathrm{A}+\mathrm{T}$ syndrome'), rats emit hyperkinetic treading to all tastes and show aversive reactions (e.g. gapes, head-shakes, chin-rubs, and forelimb-flails) to all tastes, even to normally palatable sucrose. The exact location and boundary of the striatopallidal site or sites where damage produces hyperkinetic treading has not previously been identified. Nor has it been shown how the two syndromes differ in terms of their neural damage. The present study was conducted in order to identify the crucial properties of the lesion that determine which syndrome is produced. Procedures were employed to measure the size, severity, and the neuroanatomical boundaries of the sites for the two syndromes. A novel neuron counting technique, a modification of Gundersen et al.'s 'fractionator' procedure [33] and immunocytochemical staining for glial fibrillary acidic protein were used to precisely identify these lesion sites.

\section{Materials and methods}

\subsection{Subjects}

Subjects were 47 male and 56 female Sprague-Dawley rats (Charles-River) weighing $300 \pm 50 \mathrm{~g}$ at the beginning of the experiment. They were housed singly under a 12:12 light:dark cycle. Water and food were freely available.

\subsection{Surgery}

Rats were anesthetized using first halothane (within a desiccator chamber) and then methoxy-fluorane given 
through an air anesthesia system for small animals [49]. Atropine sulfate $(3 \mathrm{mg} / \mathrm{kg}$, i.p.) and diazepam (8 $\mathrm{mg} / \mathrm{kg}$ ) were given approximately $30 \mathrm{~min}$ prior to neurotoxin injection. Bilateral lesions were made using quinolinic acid $(10 \mu \mathrm{g}$ in $1 \mu \mathrm{l})$. This neurotoxin has been suggested to play a role in the natural production of certain hyperkinetic disorders such as Huntington's disease in humans [13]. Most lesions were focused on posterior and ventral striatopallidal structures, since previous studies have indicated that lesions of these structures may have a special role in exaggerated treading $[11,19]$. Lesions intended to damage the ventral striatum or nucleus accumbens were centered at the coordinates: AP $+1.7 \mathrm{~mm}$ anterior to bregma; $\mathrm{L} \pm 1.5$ $\mathrm{mm}$ lateral to bregma; $\mathrm{V}-7.4 \mathrm{~mm}$ below the skull ( $n=8$ rats). Lesions intended to damage the neostriatum or caudate nucleus and putamen (CPU) were aimed into four different quadrants. These included the dorsolateral quadrant: AP $+1.0 \mathrm{~mm} ; \mathrm{L} \pm 3.7 \mathrm{~mm}$; and $\mathrm{V}-4.5 \mathrm{~mm}$ ( $n=8$ rats), the dorsomedial quadrant: $\mathrm{AP}+1.0 \mathrm{~mm} ; \mathrm{L} \pm 1.6 \mathrm{~mm} ; \mathrm{V}-4.2 \mathrm{~mm}(n=8)$, the ventromedial quadrant: $\mathrm{AP}+1.0 \mathrm{~mm} ; \mathrm{L} \pm 1.7 \mathrm{~mm}$; $\mathrm{V}-5.6 \mathrm{~mm}(n=4)$ or the ventrolateral quadrant: AP $+1.0 \mathrm{~mm} ; \mathrm{L} \pm 3.6 \mathrm{~mm} ; \mathrm{V}-6.6 \mathrm{~mm}(n=8)$. Lesions intended to damage the globus pallidus were centered at the coordinates: AP $-1.0 \mathrm{~mm} ; \mathrm{L} \pm 2.8 \mathrm{~mm}$; V -7.5 $\mathrm{mm}$ ( $n=13$ rats). Lesions intended to damage the ventral pallidum/substantia innominata were centered at the coordinates: AP $-1.0 \mathrm{~mm} ; \mathrm{L} \pm 2.5 \mathrm{~mm} ; \mathrm{V}-8.0$ $\mathrm{mm}(n=10)$. The actual center of the lesions varied around these coordinates. The vehicle solution was always phosphate buffered saline $(\mathrm{pH}=7.4)$. Sham operated controls received infusions of PBS alone $(1 \mu \mathrm{l}$, nucleus accumbens, $n=8, \mathrm{CPU}, n=28$, pallidal $/ \mathrm{SI}$, $n=8)$. Each infusion was made over a $3 \mathrm{~min}$ period, and the needle was left in place for an additional 5 $\min$.

Each rat was implanted with bilateral chronic oral cannulae to allow for the infusion of the taste solutions into the mouth. These cannulae (heat-flared PE-50 tubing) [27] enter the mouth lateral to the first maxillary molar and exit the head near the dorsolateral boundary of the skull, where they are anchored with skull screws and acrylic cement.

Thirty min post-surgery, a second injection of diazepam $(8 \mathrm{mg} / \mathrm{kg})$ was given to reduce potential convulsive activity. Bicillin (30,000 units, i.m.) was given to reduce the chances of post-surgical infection. All rats were given $250 \mathrm{ml}$ of cereal mash (commercial baby cereal mixed with water), 5 chow pellets and $40 \mathrm{ml}$ of water per day (5-7 p.m. feeding). Aphagia and adipsia may follow striatopallidal neurotoxin injections [50]. Intake was monitored by counting the number of pellets consumed, the approximate amount of mash eaten (e.g. 50\%), and the amount of water drank within a 24-h period. Rats were weighed daily. If a rat lost weight compared to his pre-surgical weight, then an intubation procedure was initiated. For each $5 \mathrm{~g}$ of weight lost, the rat was intubated with $12 \mathrm{ml}$ of vitamin supplemented sweetened milk solution, up to 3 intubations per day. Rats were considered aphagic if neither chow nor pellets were eaten.

\subsection{Behavioral observations}

Sensory-triggered hyperkinetic treading and other taste reactions were videotaped, after an initial habituation trial, to oral infusions of sucrose $(1 \mathrm{M})$ or quinine HCL $\left(3 \times 10^{-4}\right)$ in counterbalanced order. Sucrose elicits primarily ingestive responses and quinine elicits primarily aversive responses from normal rats. The initial trial ( $48 \mathrm{~h}$ post-surgery) used distilled water oral infusions for habituation.

A delivery tube was connected to the oral cannula, and the rat was placed in a transparent test chamber, which was suspended over an angled mirror. The mirror reflected a view of the rat's mouth and forepaws to the videocamera. Following a $5 \mathrm{~min}$ habituation period, $1 \mathrm{ml}$ of the test solution was infused directly into the mouth over a $1 \mathrm{~min}$ period. If multiple stimuli were given in a single day, there was a two hour inter-test interval. Tests were run between 4 and 8 p.m. (i.e. shortly prior to lights out). For aphagic rats receiving intubations, taste trials were conducted at least $2 \mathrm{~h}$ after the last intubation.

\subsection{Taste reactivity scoring}

Videotapes were scored in slow motion (frame by frame to 1/10 normal speed) for the occurrence of exaggerated paw treading. A stringent criterion was used for the analysis of paw treading in order to avoid 'false positive' scoring of normal treading and ensure that only pathological exaggerated paw treading would be included. A unit of paw treading was required to be at least 5 continuous seconds of rhythmic forward and backward extension of the forepaws $180^{\circ}$ out of phase, with the two paws extended at least $1 \mathrm{~cm}$ apart in every cycle, at a frequency of roughly $3.5 \mathrm{~Hz}$.

Videotapes were also scored for the occurrence of aversive reactions and for positive hedonic reactions. Aversive actions include: gapes: large openings of the mouth and jaw lasting approximately $125 \mathrm{~ms}$; chin-rubbing: bringing the chin in direct contact with the floor and projecting the body forward; face-washing: either a single wipe with the paws over the face or a bout of several wipes; forelimb flails: shaking of the forelimb back and forth; and rapid head-shaking. Paw treading was specifically excluded from the aversive scores to reduce artificial aversive score inflation. 
The following positive reactions were scored: lateral tongue protrusions (nonrhythmic) lasting about $160 \mathrm{~ms}$; rhythmic tongue protrusions: these protrude along the midline with a cycle length of roughly $160 \mathrm{~ms}$; and paw-licking. Each action was scored and counted using criteria described in Grill and Norgren [26].

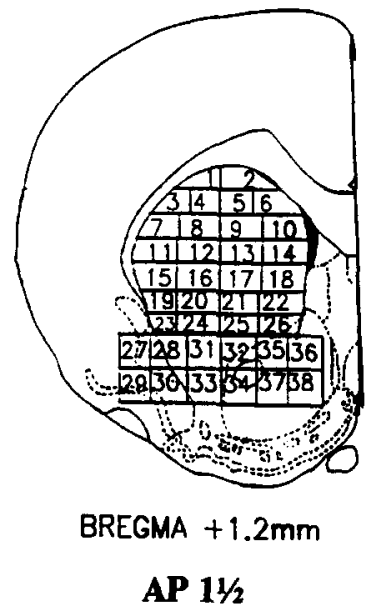

AP $11 / 2$

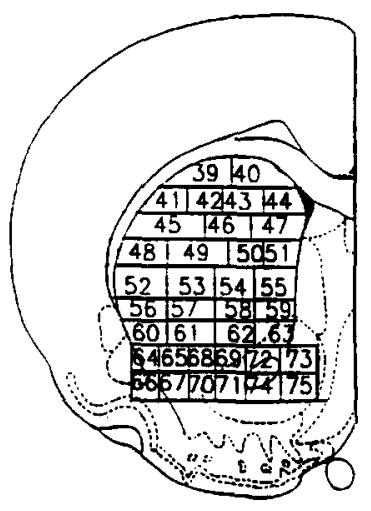

BREGMA $+0.7 \mathrm{~mm}$

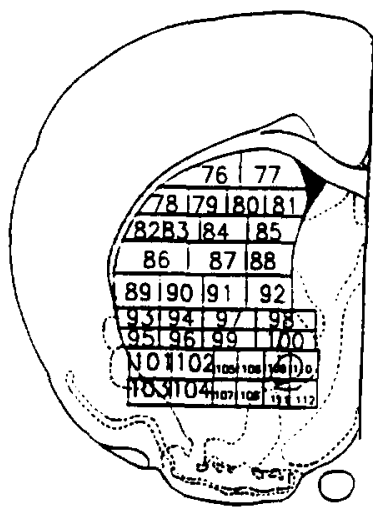

BREGMA $+0.2 \mathrm{~mm}$

AP 2

AP 21/2

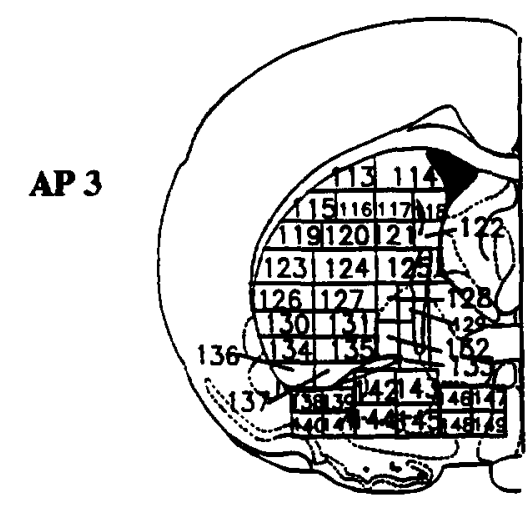

BREGMA $-0.3 \mathrm{~mm}$

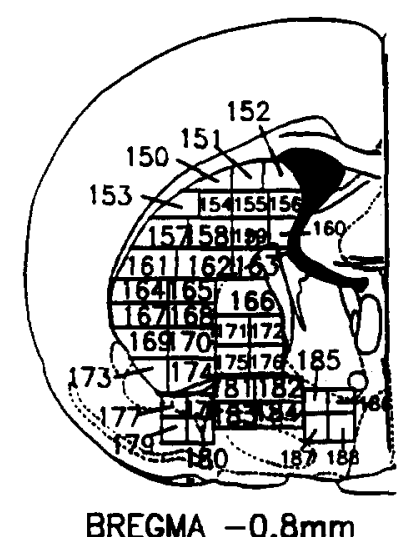

AP 31/2

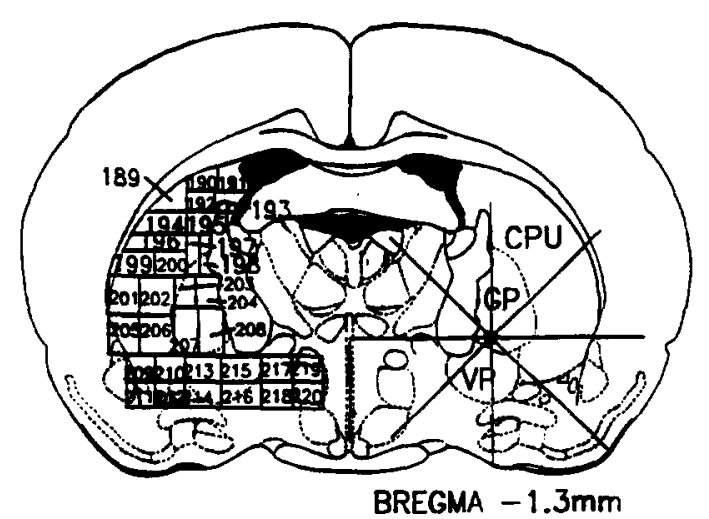

AP 4

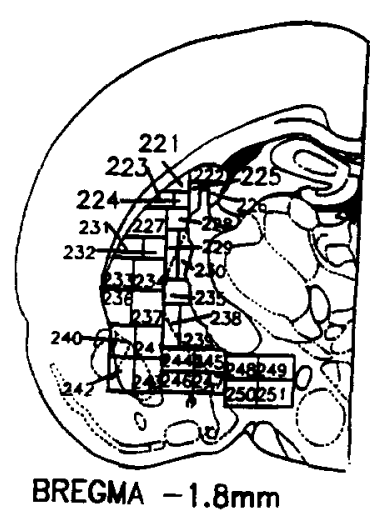

AP 4 $4 \frac{12}{2}$

Fig. 1. Fraction zones. The 251 fraction zones used for the fractionator technique. Each fraction is labeled by its assigned number. Smaller fractions are positioned around the excitotoxin microinjection targets. Lower left: example of lesion boundary analysis along 8 radial arms from the center of a lesion. Neuron counts were continued along each arm until either the boundary of the lesion edge or the circumference was reached $(2.5 \mathrm{~mm}$ from center, whichever came first. 


\subsection{Histology}

At the conclusion of the behavioral testing, each rat was deeply anesthetized and intracardially perfused. Perfusions were completed with $0.9 \%$ saline followed by $10 \%$ formalin in PBS $(\mathrm{pH}=7.4)$. Brains were removed and stored in a $30 \%$ sucrose solution (10\% formalin). The brains were blocked, frozen, and sliced into $30 \mu \mathrm{m}$ slices using a sliding microtome. Slices were mounted directly onto gelatin-coated slides for Cresyl violet staining. The slides were dipped in xylene and ethanol baths $(70 \%, 95 \%$ and $100 \%)$ for cleaning and defatting. After being dipped in Cresyl violet, the slides were taken through the final alcohols and xylenes prior to coverslipping using permount. A modification of Gundersen's fractionator technique for obtaining accurate regional counts of neurons was completed in order to specify the exact borders and intensity of damage within this general region.

\subsection{Approximate localization of damage}

An initial examination of each brain was made at 5 anterior-posterior levels (Bregma $+1.7 \mathrm{~mm},+0.7 \mathrm{~mm}$, $-0.3 \mathrm{~mm},-1.3 \mathrm{~mm}$, and $-2.3 \mathrm{~mm}$ ) in order to identify the approximate location of lesions in the neostriatum, globus pallidus, ventral pallidum or substantia innominata. Neuron counts were made using a light microscope (Leitz Laborlux $\mathbf{S}$ ), a videocamera and a computerized videoimage analysis system (JAVA, Jandel) in nine regions at each level. The videoimage analysis system was used to project an image of the slice upon a monitor and exactly delineate the borders of the particular regions within the striatopallidal system. This technique led to a high consistency in terms of the region shapes and positions between the different rat brain slices. To determine the neuronal density within striatopallidal regions, neuron cell counts were completed using a eyepiece reticule $(10 \mathrm{~mm}$ by $10 \mathrm{~mm}$ divided into $1.0 \mathrm{~mm}$ squares) placed into a $10 \times$ eyepiece. The light microscope analysis was completed using a $20 \times$ objective (total magnification $200 \times$ ). A neuron was defined for scoring purposes as an intact circular or ovoid shaped object with a light purple interior stain, at least 10 microns in diameter (one-fifth of a 50 micron length individual grid). Both medium (10-15 microns in diameter) and large ( $>15$ microns in diameter) neurons were counted. The nucleus was not required to be present in order for an object to be counted as a neuron.

\subsection{Fractionator analysis of lesion boundaries}

Identified lesions were analyzed in detail using our modification [19] of the fractionator technique [33].
Twenty-five rats were selected for the modified fractionator technique $(8$ controls, 17 hyperkinetic paw treaders). This procedure was conducted in 3 stages.

Stage 1. Normal reference maps. First, in order to obtain accurate normal baseline neuron averages, the normal neuronal density of 251 brain fractions from 7 AP levels was determined using a modification of Gundersen's fractionator technique [33] (see Fig. 1). Fractions ranged in cubic volume from $0.03 \mathrm{~mm}^{3}$ to 0.375 $\mathrm{mm}^{3}$. The smallest fractions were grouped around sites of injections. For a complete listing of the normal neuron density for all fractions $(n=251)$ of the striatopallidal system see Table 1 .

For each fraction, an exhaustive neuronal count was made of a $250 \times 250 \times 30$ micron 'core sample'. This was done by delineating a 250 square micron area of a 30 micron tissue section (randomly chosen within each fraction) at $400 \times$ magnification using the videoimage analysis system. Neurons were counted throughout the total volume of the fraction by counting all neurons at each plane of focus. Neuronal density varied from fraction to fraction in normal animals from 12 neurons in fractions from the globus pallidus to 154 neurons in fractions from the ventrolateral striatum. However, neuronal density varied by less than $25 \%$ between different rats for any given fraction. This consistency between animals in neuronal density for a given fraction allowed us to set a criterion for the detection of lesions.

Stage 2a. Identifying lesions by the fractionator method. 'Moderate neuron loss' was judged to exist if a fraction had less than $50 \%$ of the normal mean number of neurons for that fraction. Since normal brains vary by less than $25 \%$, a neuronal density decrease below $50 \%$ falls well beyond the normal range. Areas that had greater than $50 \%$ neuron loss but less than $80 \%$ neuron loss were labeled as the shell of the lesion. 'Severe neuron loss' existed if the fraction had lost at least $80 \%$ of its neurons compared to the normal mean. Fractions which had the most severe neuron loss were labeled as the core of the lesion.

Stage $2 b$. Lesion border analysis. Once the center of the lesion had been located, 8 radial arms were drawn using the video image analysis system (JAVA System, Jandel, Corp.) emanating from this center along the major compass points $\left(0^{\circ}, 45^{\circ}, 90^{\circ}, 135^{\circ}\right.$ etc., Fig. 1 lower left). Core sample counts were taken along each line at 250 micron steps at $400 \times$ magnification (see above) until the neuron density rose above $50 \%$ of the normal level for that fraction. Lesions borders were mapped onto stereotaxic atlas pictures of the representative brain slices [63] and were traced into the computer using a digitizing tablet and a 3 -D reconstruction program (PC3D, Jandel). The digitized slices were converted into a 3-D representation for lesion viewing using a lateral, ventral, and a dorsal 3-D view. 
Stage 3. Subtraction of noncrucial sites of damage. A composite map of shared damage was made for each syndrome group. This was done by first adding the mapped lesions of each rat that showed a particular syndrome together. Then any subregion of damage that was not shared unanimously by all brains within the group was subtracted away in order to eliminate areas of damage that were not necessary to produce the syndrome. The remaining composite lesion identified the common site that was damaged in every rat which displayed either hyperkinetic treading plus aversion or hyperkinetic treading alone.

\subsection{GFAP-immunoreactivity}

In order to confirm lesion placement by an independent measure, a second technique was employed that mapped regions of gliosis based on glial fibrillary acidic protein immunoreactivity (GFAP-IR). Glial fibrillary acidic protein immunoreactivity preferentially stains reactive astrocytes. Free-floating 30 micron sections were rinsed in three consecutive washes (PBS with 1\% bovine serum albumin and $0.03 \%$ triton $\times 100$ ). The slices were transferred to $1.5 \mathrm{ml}$ centrifuge tubes containing rabbit anti-GFAP (primary, diluted 1:500, Dako Corp.) and placed on a roto-torque to turn slowly for $20-24 \mathrm{~h}$ at $5^{\circ} \mathrm{C}$. The slices were rinsed in three consecutive PBS-BSA $(0.03 \%$ triton $\times 100)$ washes and placed into $1.5 \mathrm{ml}$ centrifuge tubes containing peroxidase conjugated to goat anti-rabbit IgG (secondary, diluted 1:100, Dako Corp.) and rotated for $1 \mathrm{~h}$ at room temperature. The slices were taken through a final set of rinses (PBS-BSA $+0.03 \%$ triton $\times 100$ ) followed by an $8 \mathrm{~min}$ bath in freshly prepared 3,3-diaminobenzidine tetrahydrochloride on a shaker. The slices were then rinsed in distilled water, mounted directly onto a slide (gel-coated $2 \times$ ), defatted and coverslipped using permount.

Areas of lesion-induced gliosis are much darker than the surrounding area after GFAP immunoreactive staining. Using a computerized densitometry video analysis based on pixel darkness (shades of grey from light grey to black) the reactive gliosis was calibrated, and a criterion of the darkest $20 \%$ of the pixels and the darkest $10 \%$ of the pixels was set and used to identify gliosis shell and core regions respectively. The gliosis shell and core were traced on to pictures of brain slices [63]. The lateral ventricle and corpus callosum were used as landmarks. Maps of lesions constructed by gliosis analysis were compared to maps constructed by neuronal density analysis.

\subsection{Statistics}

Group averages for taste reactions and paw treading duration were compared using the Mann-Whitney $U$ - nonparametric test. Mean neuron counts for each striatopallidal region were compared across groups by a two factor (region $\times$ treatment group) analysis of variance (ANOVA). Differences across regions within lesion groups were analyzed by the a posteriori Newman-Keuls test. Means of regional neuron counts between lesion groups were also compared using the Newman-Keuls test.

\section{Results}

\subsection{Behavioral results}

Nine of eleven rats that received bilateral neurotoxin injections into the GP displayed hyperkinetic treading to quinine alone ( $\mathrm{T}$ Syndrome). The average treading duration in this group was $14 \mathrm{~s}$ per quinine taste trial (compared to $0 \mathrm{~s}$ for controls, Mann-Whitney test, $P<0.05$, see Fig. 2). No rat in this group showed treading to sucrose taste infusions. Taste reactions (besides exaggerated treading to quinine) did not differ from controls, and these rats ate and drank normal amounts.

Eight of twelve rats that received bilateral neurotoxin injections into the ventral pallidum/substantia innominata showed hyperkinetic treading to both quinine and sucrose solutions. These same rats $(\mathrm{A}+\mathrm{T}$ Syndrome) also had a significant increase in the number of aversive responses [gapes, head-shakes, forelimb-flails and chin-rubbing] to sucrose (1 M) compared to sham-injected control rats, which showed

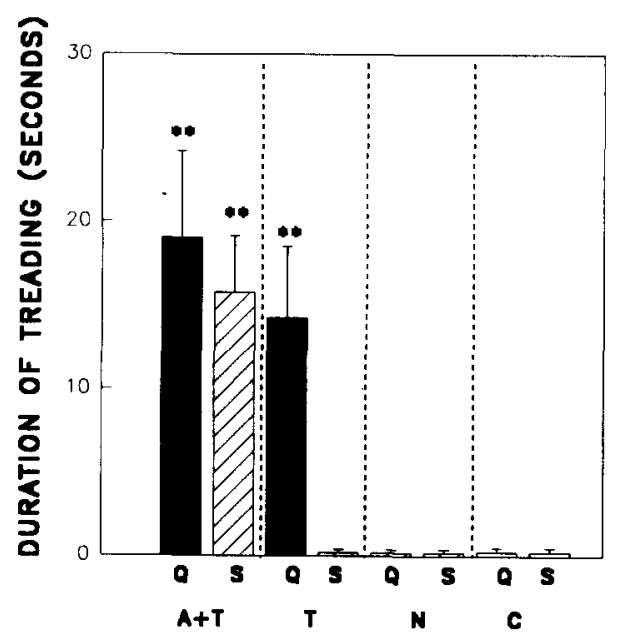

Fig. 2. Duration of hyperkinetic treading to either quinine $(Q)$ or sucrose (S) oral infusions by: (1) rats that show the combined syndrome of exaggerated treading plus enhanced aversion $(A+T$ Group); (2) rats that showed only the exaggerated treading syndrome without enhanced aversive taste reactivity (T Group); 3) rats with lesions that did not show either treading or enhanced aversion (N); and (4) sham-injected controls (C). * and * denote significance at the $P<0.001$ and $P<0.05$ levels, respectively. 
none. The average treading duration for this group was $19 \mathrm{~s}$ for quinine and $16 \mathrm{~s}$ for sucrose (Mann-Whitney test, $P<0.01$ compared to $0 \mathrm{~s}$ for controls, see Fig. 2). The number of aversive responses to sucrose was increased to an average of 13.5 compared to 0 for controls (Mann-Whitney test, $P<0.001$ ). Rats that showed enhanced aversion plus hyperkinetic treading (A $+\mathrm{T}$ Syndrome) were also aphagic and adipsic following surgery. The average length of aphagia and adipsia was 5 days (range, 2-7 days for these rats). The average weight loss was $64 \mathrm{~g}$. General motor activity appeared suppressed and some rats were severely aki-
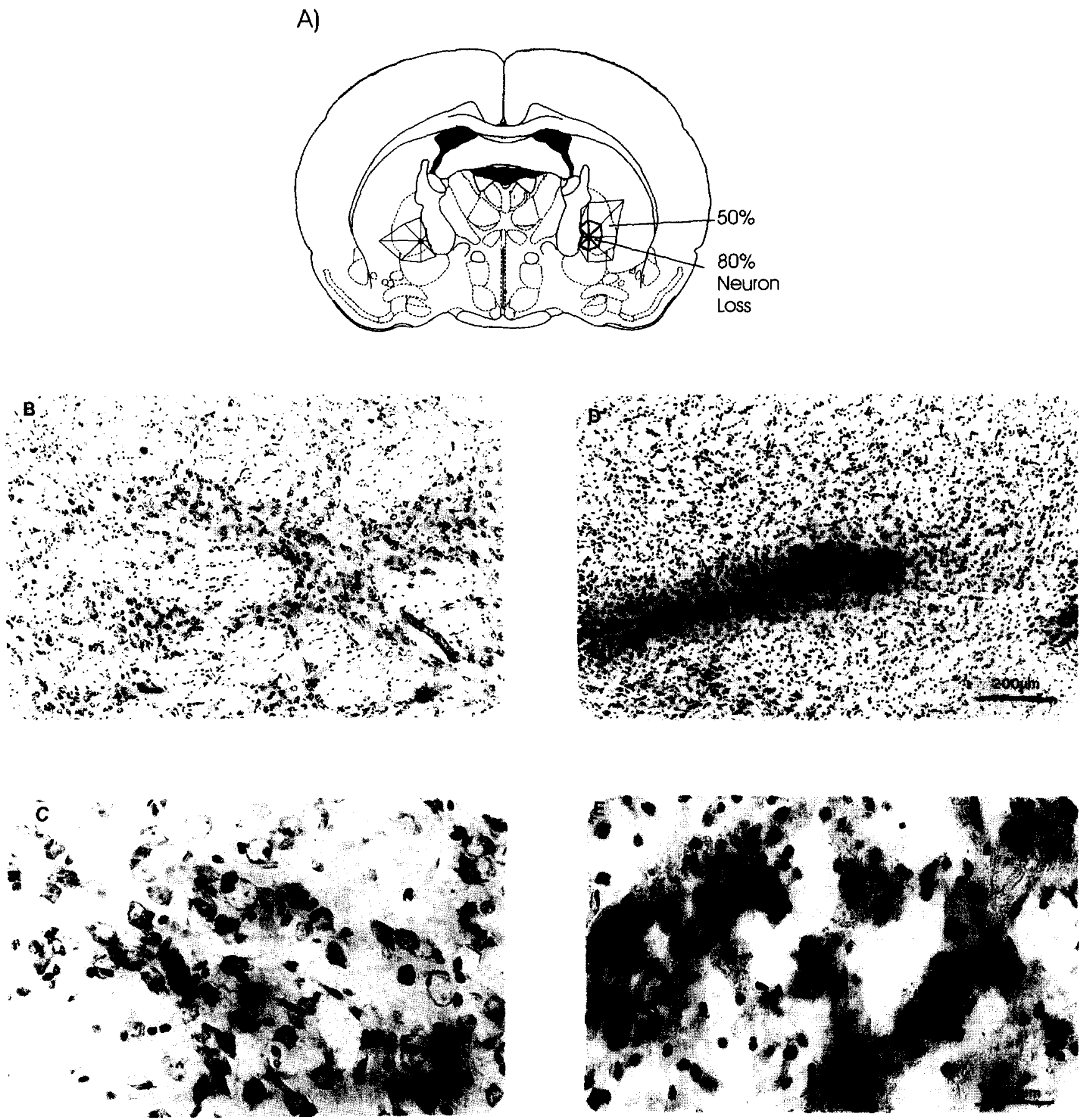

Fig. 3. T Group lesion. Representative lesion mapped to show the result of the fractionator and boundary analysis techniques (A), photomicrograph of the globus pallidus from a control rat $(10 \times$ magnification, B), and high magnification photomicrograph of the globus pallidus from a control rat $(40 \times, C)$, photomicrograph of lesion from a T Group rat $(10 \times, D)$ and a high magnification photomicrograph of the lesion core from a T Group rat $(40 \times, \mathrm{E})$. 
netic with postural deviations such as chronic limb extension.

Rats that received neurotoxin injections into either the ventral striatum or into any quadrant of the neostriatum did not show hyperkinetic treading or changes in taste reactivity ( $\mathrm{N}$ Group).

\subsection{Globus pallidus lesions: production of treading alone ( $T$ syndrome)}

Lesion histology was completed 4-14 days postsurgery for the T-Group rats. In an analysis to detect shrinkage, measurements for striatal or pallidal width and length were similar for the T-Group rats and controls. This finding suggests that there was minimal shrinkage due to excitotoxic injury. Reactive astrogliosis was prominent in the lesion area and most likely filled in the regions of neuronal loss. For the T-Group rats with neurotoxin injections aimed at the GP that displayed hyperkinetic treading to quinine alone, significant bilateral damage compared to sham-injected controls was noted in only two regions: the globus pallidus and the ventrolateral neostriatum (Three-way ANOVA (Group $\times$ Region $\times$ AP level), $\mathrm{F}(104,712)=$ 4.806, $P<0.001$; globus pallidus and ventrolateral neostriatum regions were identified by post-hoc comparisons with control group, $P<0.05$, Newman-Keuls). Both regions had bilateral damage located at Bregma $-0.3 \mathrm{~mm}$ and $-1.3 \mathrm{~mm}$ (globus pallidus $\geq 67 \%$ bilateral damage; ventrolateral neostriatum $\geq 40 \%$ neuron loss). No other regions distal from the injection site had bilateral damage.

In the more detailed fractionator analysis, a rat-byrat examination was completed for neuron loss within the globus pallidus and ventrolateral neostriatum for the rats that showed the hyperkinetic treading to quinine alone. Each rat in this group had damage in a subregion of the globus pallidus (Fractions numbers 208 and 207; Fig. 3). The boundary analysis using neuron counts along the 8 radial arms emanating away from the center of the lesion for each rat, showed that each rat had damage in the ventromedial corner of the GP, immediately lateral to the internal capsule and dorsal to the VP/SI region (minimum damage $=60 \%$ bilateral neuron loss for each rat, see Fig. 4). The stereotaxic center for this crucial site, determined by subtraction analysis, was located at $1.3 \mathrm{~mm}$ posterior from bregma (at this AP level each rat had the largest lesion area). The lateral diameter of the common site was $0.8 \mathrm{~mm}$, the dorsoventral diameter was $1.0 \mathrm{~mm}$ and the AP diameter was $1.0 \mathrm{~mm}$.

Surrounding subregions of the GP were damaged in only some rats that showed the hyperkinetic treading to quinine. Eight out of 9 rats had $>50 \%$ damage to the overlying dorsal GP and 6 out of 9 rats had $>50 \%$ neuron loss in the lateral GP. Outside of the GP, the
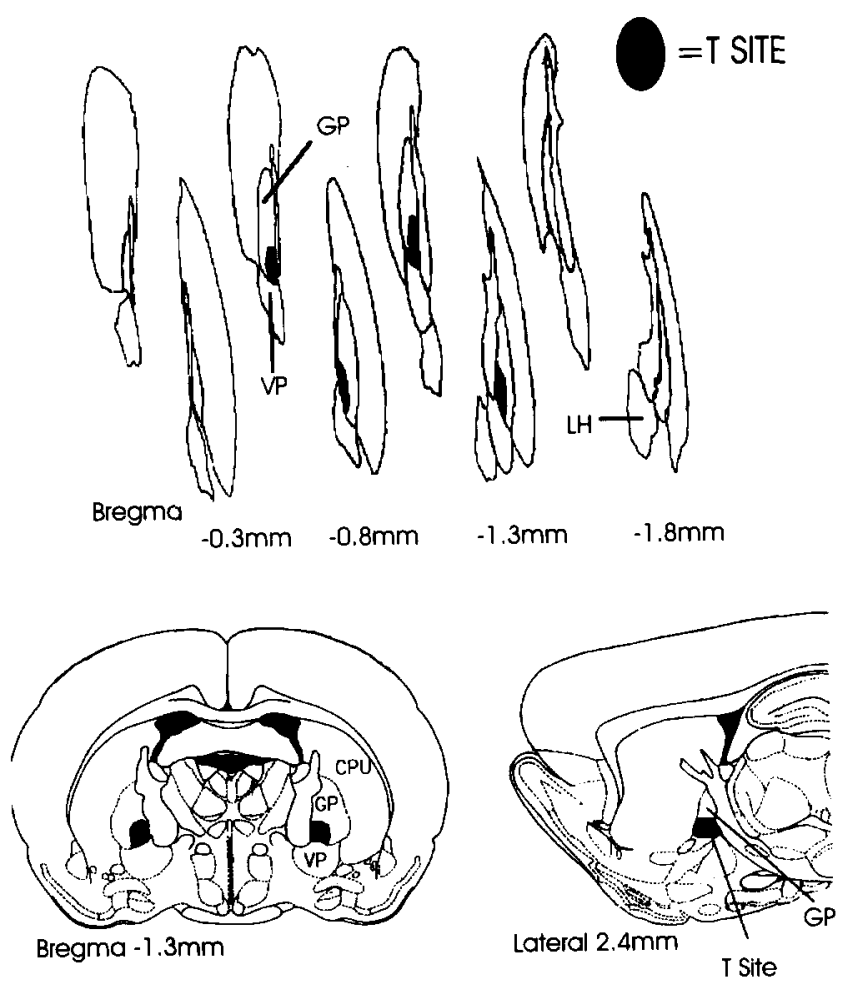

Fig. 4. Crucial site for the $T$ syndrome as mapped by our modified fractionator procedure. The same views are shown as used in Fig. 6. Solid region denotes site where all rats had at least $60 \%$ neuron depletion.

ventrolateral neostriatum was bilaterally damaged in 2 out of the 9 rats. Ventral to the GP, the VP/SI or LH was bilaterally damaged in only 1 out of 9 rats. GFAPIR had the darkest pattern of staining over the ventromedial GP for each of the rats showing hyperkinetic treading to quinine alone. This pattern of results supports the idea that damage to the ventromedial GP is sufficient to produce the hyperkinetic treading syndrome ( $\mathrm{T}$ syndrome).

Significant GP damage was not seen in the nonsymptomatic rats $(n=2)$ that received injections aimed at the GP. Unilateral damage of the GP was noted in both rats.

\subsection{Ventral pallidum/substantia innominata lesions: production of treading plus aversion}

Lesion histology for the A + T Group rats was completed between 4 and 15 days post-lesion. In an analysis to detect shrinkage, measurements of striatal and pallidal lengths and widths were similar between $A+T$ Group rats and sham injected controls. This finding suggests that the total volume of the striatopallidal region was not significantly different between these two groups and that accurate neuronal counts for both regional and fractionator analyses could be obtained. $\mathrm{A}+\mathrm{T}$ Group rats had significantly more damage in the 
$\mathrm{VP} / \mathrm{SI}$ region at Bregma $-1.3 \mathrm{~mm}$ compared to both control rats and T Group rats $(81 \%$ average depletion, Three-way ANOVA (Group $\times$ Region $\times$ AP level) $F(104,712)=4.806, P<0.001$; ventral pallidal region identified by post-hoc comparisons with controls and with T Group rats, $P<.0 .05$, Newman-Keuls). Com- pared to only sham-injected controls the $A+T$ Group rats also had significant bilateral neuron loss in the GP (34\% neuron depletion, $P<0.05$, Newman-Keuls) and in the LH $(59 \%$ neuronal depletion, $P<0.05$, Newman-Keuls). No other distal regions had bilateral neuronal damage.
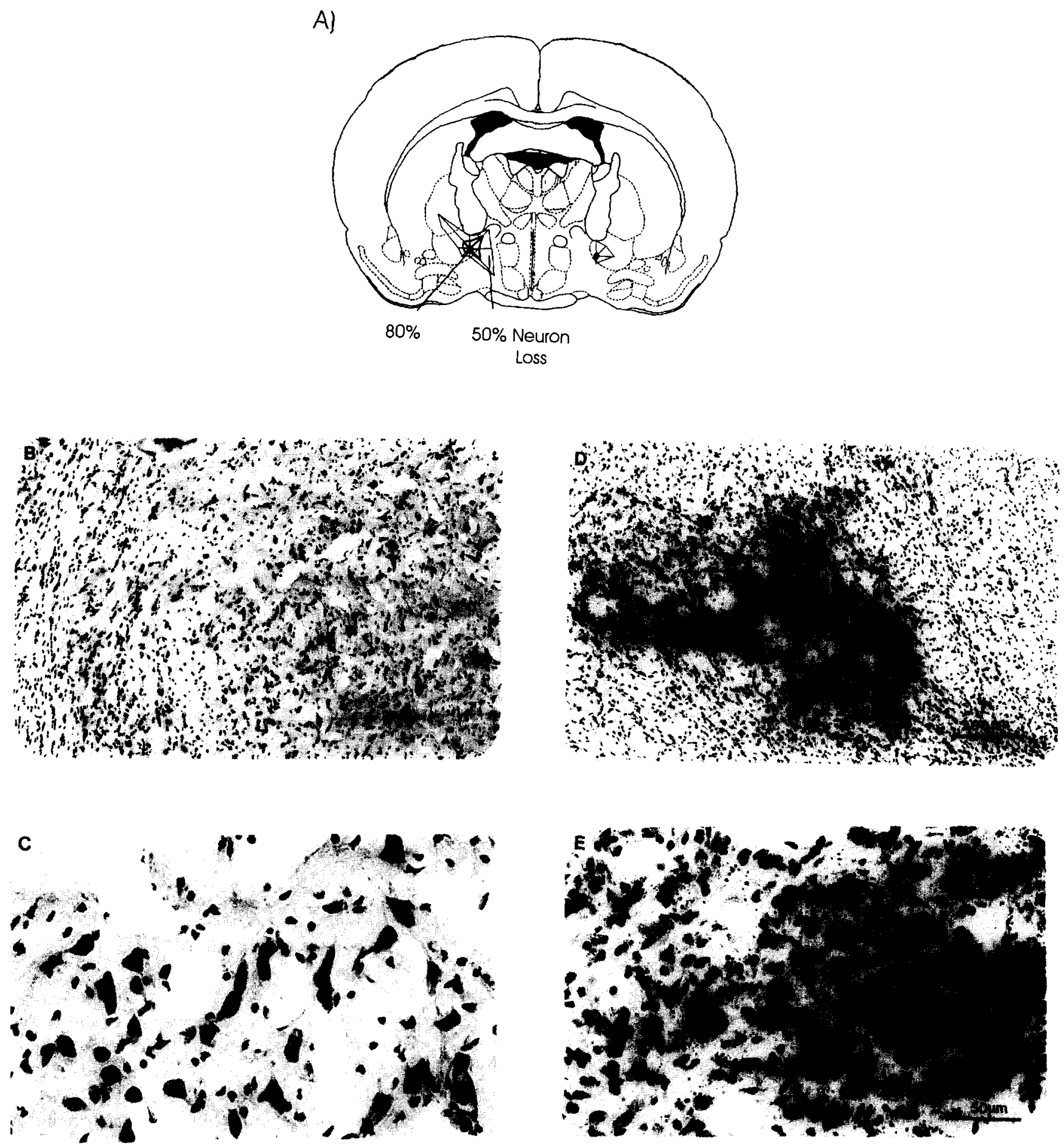

Fig. 5. A + T Group lesion. Representative lesion mapped to show the result of the fractionator and boundary analysis techniques (A), control photomicrographs of the ventromedial ventral pallidum/substantia innominata (B and C, same magnifications as in Fig. 3), low magnification (D) and high magnification $(E)$ of a lesion from a rat in the $A+T$ Group. 

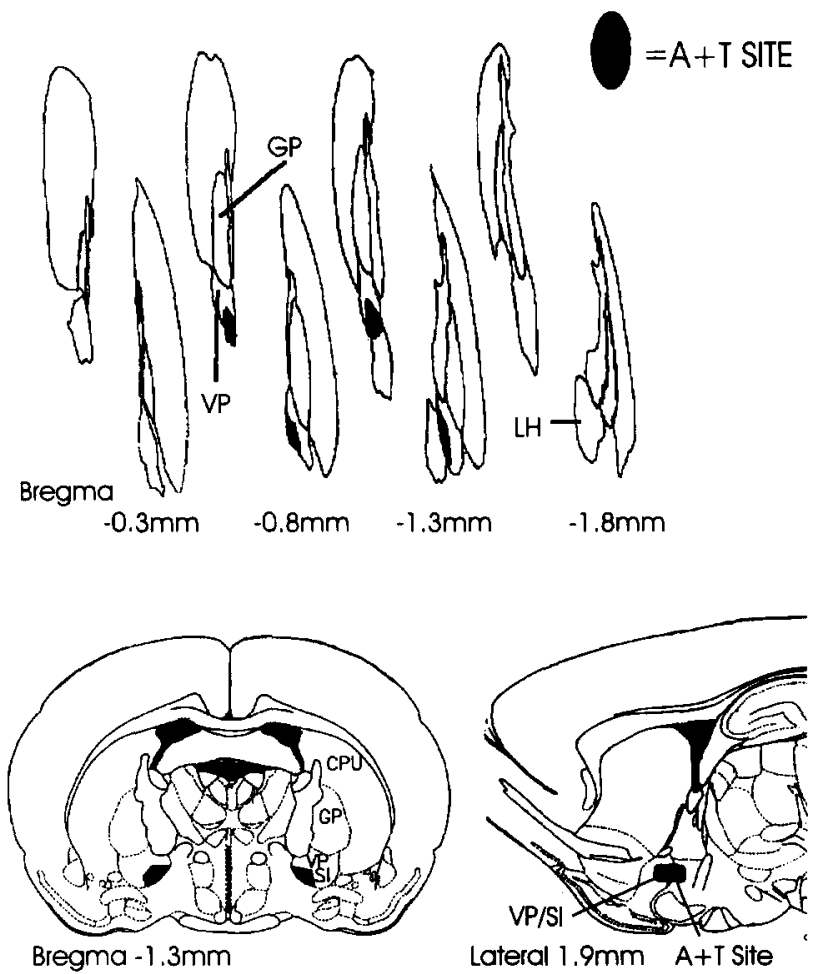

Fig. 6. Crucial site for the $A+T$ syndrome. A 3-dimensional view (above) and coronal and sagittal 2-D views (below) of the 'necessary and sufficient' site of damage, as mapped by our modified fractionator procedure, that produces both hyperkinetic treading and an enhancement of aversive reactions to oral infusions. Solid region denotes site where all rats had at least $70 \%$ neuron depletion.

Results from the more detailed fractionator analysis of striatopallidal subregions showed that common damage was located only in a subregion of the VP/SI in the $\mathrm{A}+\mathrm{T}$ Syndrome group (Fraction numbers 215 and 216; Fig. 5). The lesion boundary analysis using the 8 radial arms emanating from the lesion center for each rat showed that the common subregion within the VP/SI for the group was the ventromedial corner which is adjacent to, but outside of, the dorsolateral rim of the lateral hypothalamus and ventral and medial to the globus pallidus (see Fig. 6). Each rat had at least $70 \%$ neuronal depletion within this area of the VP/SI. The center for this common site was $1.3 \mathrm{~mm}$ posterior from bregma, $2.0 \pm 0.5 \mathrm{~mm}$ lateral from bregma, and $7.3 \pm 0.25 \mathrm{~mm}$ ventral to the skull as depicted in the stereotaxic atlas of Paxinos and Watson, 1986. The lateral diameter of the shared lesion site was $1.0 \mathrm{~mm}$, the dorsoventral diameter was $0.5 \mathrm{~mm}$ and the AP diameter was $1.0 \mathrm{~mm}$.

Subregions outside of the ventromedial VP/SI were damaged in some but not all rats that showed both enhanced aversion plus hyperkinetic treading $(A+T$ Syndrome). Six out of 8 rats had $>50 \%$ neuronal depletion bilaterally in the adjacent dorsal ventral pallidum and 4 out of 8 rats had $>50 \%$ bilateral neuron loss in the lateral VP/SI. Outside of the VP/SI, the GP had $>50 \%$ bilateral neuron loss in 5 out of 8 rats, and ventrally, the LH had $>50 \%$ neuron loss in 4 out of 8 rats. No other structure was damaged in at least 4 of the rats. Dense GFAP-IR was measured over the ventromedial VP/SI in each rat that displayed the enhanced aversion plus hyperkinetic treading. These results support the conclusion that damage to the ventromedial VP/SI is sufficient to produce these behavioral changes.

Four rats that received injections aimed into the VP/SI did not show any behavioral changes and even though they had some bilateral neuron loss in the VP / SI compared to controls. These 'non-symptomatic' rats had much less severe damage compared to 'symptomatic' rats in the VP/SI (20-59\% neuron loss in the non-symptomatic rats compared to a minimum of $70 \%$ bilateral neuron loss in each symptomatic rat). No non-symptomatic rat had bilateral damage exceeding $70 \%$. This result indicates that a threshold of neuronal depletion is necessary for the production of the enhanced aversion and hyperkinetic treading syndrome.

\subsection{Other lesion groups}

Rats that received injections aimed at the ventral striatum had the most damage in this region $(>65 \%$ bilateral neuron loss). Rats that received neurotoxin injections into the different neostriatal quadrants had significant bilateral damage in those respective quadrants (dorsolateral neostriatal injection group had > $69 \%$ bilateral neuron loss in the dorsolateral neostriatum; dorsomedial neostriatal injection group had > $62 \%$ bilateral neuron loss in the dorsomedial neostriatum; ventrolateral neostriatal injection group had > $75 \%$ bilateral neuron loss within the ventrolateral neostriatum; and ventromedial neostriatum injection group had $>81 \%$ neuron loss in the ventromedial neostriatum). Any extra-striatal damage was seen unilaterally in the striatal lesion groups.

\section{Discussion}

Damage to two neuroanatomically separate sites was found to produce the two different behavioral syndromes of hyperkinetic treading originally differentiated by Berridge and Cromwell [11]. Lesions of the ventromedial third of the globus pallidus produced the sensory-triggered hyperkinetic treading syndrome alone (T Syndrome) but did not produce enhanced aversion to food (see Fig. 4). This T syndrome site is $1.0 \mathrm{~mm}$ by $0.8 \mathrm{~mm}$ by $1.0 \mathrm{~mm}$ in diameter and lies just lateral to the internal capsule and dorsal to the VP/SI (Fractions 207 and 208). Lesions of the ventromedial corner of the VP/SI produced hyperkinetic treading plus an 
enhancement of aversive taste reactions to oral infusions (A + T Syndrome; for more discussion of 'aversion sites' see Cromwell and Berridge [19]). The crucial site appears for the $A+T$ syndrome to be $1 \mathrm{~mm}$ by 0.5 $\mathrm{mm}$ by $1.0 \mathrm{~mm}$ in diameter and is located ventral and medial to the globus pallidus and immediately lateral to the border of the lateral hypothalamus (Fractions 215 and 216; Fig. 6).

The site of damage (rather than the diameter or the suprathreshold severity of a lesion) appears to be the primary determinant of which treading syndrome is produced. The two sites are approximately $0.5 \mathrm{~mm}$ apart. Lesions that produce treading plus enhanced aversion are ventral and medial to the lesions that produce the hyperkinetic treading alone, but they need not be larger or more severe than lesions that produce the treading syndrome alone. (so long as bilateral damage in the vmVP/SI, Fractions 215 and 216 in Fig. 3 exceeds $>60 \%$ neuron loss). Interestingly, ventral pallidal lesions that produce the combined syndrome need not damage the globus pallidus ' $T$ Site'. However, lesions that do damage both sites always produce the combined syndrome of exaggerated treading to both sucrose and quinine, as well as aphagia and enhanced aversive taste reactions.

\subsection{Ventromedial GP subregion: anatomical connectivity}

The globus pallidus has been divided into ventromedial and dorsolateral sectors based upon differential neuroconnectivity of the sectors with other structures. The neostriatum or caudate nucleus and putamen (CPU) are the primary afferents to both sectors [38,61]. The subthalamic nucleus is the primary efferent $[58,59]$. The ventromedial GP, where lesions produce hyperkinetic treading to quinine, receives unique input from the ventromedial caudate nucleus and putamen (vmCPU) [60,61], which in turn receives its primary input from limbic structures: the hippocampus, amygdala, and cingulate cortex $[24,51,61]$. The ventromedial GP also receives direct input from the nucleus accumbens, which is part of the 'limbic' ventral striatopallidal system [43,53]. Injections of the GABA antagonist, picrotoxin, into the ventromedial GP abolish hyperlocomotion initiated by stimulation of the nucleus accumbens [43]. This direct influence of the ventral striatum upon the ventromedial globus pallidus has been argued to provide "a direct link between limbic and motor systems for the translation of motivation into action." (Jones and Mogenson, [43] p. 104.)

\subsection{Ventromedial VP / SI subregion: anatomical connec- tivity}

Both the $\mathrm{A}+\mathrm{T}$ and $\mathrm{T}$ hyperkinetic syndromes have been shown to involve an interaction of sensorimotor and motivational (aversion) processes [11]. The T Syndrome is activated by naturally aversive stimuli, such as quinine, whereas the $\mathrm{A}+\mathrm{T}$ Syndrome causes all tastes to be reacted to as aversive. Both the ventromedial VP/SI and the ventromedial GP have limbic neuroanatomical connections that make them distinctively different from dorsolateral subregions of the VP and GP [34-37,40,55,79]. Several researchers have suggested that the ventromedial subregions of the ventral pallidum/substantia innominata and globus pallidus have a special role in motivational and motor interaction $[5,53,62,79]$.

The ventromedial VP/SI has a set of distinct connections, which for the most part are different from those of the dorsolateral VP/SI $[29,30,39,78]$. Specifically, the ventromedial VP/SI [28] receives input from the shell portion of the nucleus accumbens [41,78], the medial part of the bed nucleus of the stria terminalis (mBNST), the medial nucleus of the central amygdala (ceAM), the medial preoptic nucleus (MPO), the anterior hypothalamus (aHY), the medial area of the lateral hypothalamus (LH) and the ventromedial hypothalamus (VMH) [32]. This input to the ventromedial VP/SI from various limbic structures has reinforced the idea that ventral striatopallidal efferents participate in motivational processing $[5,39,60]$.

The ventromedial VP/SI sends reciprocal connections back to the mBNST, ceAM, MPO, aHy, and $\mathrm{LH}$ [9,31]. Efferent projections also extend to the pedunculopontine nucleus (PPnT), the cortex, and the ventral tegmental area (VTA) [36,76,77]. Several researchers have proposed that the VP projections to either cortex or to the PPnT could allow access to sensorimotor processes $[36,54,56]$. The VP to PPnt connection has been shown to influence locomotion by experiments that used both electrophysiological and pharmacological techniques $[52,54,55]$. The ventral pallidal projection to the VTA region may also influence motor circuitry by modulating the dopamine neurons that project to the 'motor' dorsal striatum [30]. This ventral to dorsal striatopallidal connection through the midbrain could be important in integrating motivational and motor processes.

\subsection{Neuropathological models and clinical perspectives}

Recent models of hyperkinetic movements in humans postulates that disinhibition of the lateral globus pallidus (LGP) by degenerative striatal disease is the cause of the hyperkinetic movements of certain disorders $[1,21,23,64,75]$. Disinhibition of the LGP $[3,4]$ is hypothesized by these models to be a result of a selective early degeneration of the co-localized GABA/ENK inhibitory striatopallidal projection. The 
early loss of this projection [2] is consistent with postmortem studies of brains of patients with Huntington's disease [65]. Since the LGP is thought to normally inhibit the subthalamic nucleus (STN), then, "the ultimate effect would be loss of the STN-derived excitatory drive to the MGP and $\mathrm{SNr}$ and disinhibition of the VA/VL/MD/CM-pf thalamocortical projection." (Albin et. al., [1] p. 370), resulting in hyper-excitation of the motor cortical areas and hyperkinesis.

Based upon these models, it could be predicted that pallidal destruction should lead to hypokinesis. The results of our lesion analysis, however, show that damage to the ventral pallidum/substantia innominata and globus pallidus can produce hyperkinetic treading movements triggered by oral sensory stimulation. Several factors could lead to this discrepancy between our results and the predictions made by these models of hyperkinetic disorders. The time progression and the selectivity [74] of neuropathology may be important in producing the differences between our results and the model's predictions. Human disorders usually are chronic diseases characterized by degeneration of a gradual nature. In contrast, our pathology was caused by an acute injection of excitotoxin that produced rapid onset of hyperkinetic symptoms. Acute injections of neurotoxic quinolinic acid appear to cause non-selective striatal neuron loss [12] (although this point is still controversial) [8]. More realistic techniques for mimicking Huntington's disease neuropathology have recently been devised, which use chronic injections (3-7 weeks) of smaller amounts of quinolinic acid [7,72]. For example, Bazzett and colleagues have found selective neuronal degeneration patterns within the dorsal striatopallidal system of rats after a chronic infusion of minute amounts of quinolinic acid $(3.3 \mu$ moles), not unlike that found in the post-mortem brain of a Huntington's disease patient [7]. A better test of the hyperkinetic disorder model $[1,20,64,66,75]$, would be to combine chronic excitotoxin infusions into the striatum or globus pallidus with our behavioral test of sensorytriggered hyperkinetic treading.

In any case, our results indicate that hyperkinetic movements can be elicited from rats after pallidal damage. Could damage to the pallidal region be involved in human hyperkinetic disorders? Lange and colleagues found that the volume of the lateral globus pallidus was reduced in Huntington's chorea as much as the caudate nucleus and putamen (57\% globus pallidal reduction compared to $56 \%$ striatal reduction) [47] This finding prompted Lange et.al. to note that, "In Huntington's chorea the pallidum was more severely affected than is commonly appreciated." (Lange et al., [47] p. 401).

Do hyperkinetic symptoms ever occur following lesions to the pallidal region without striatal damage in humans, as would be predicted from our observations in rats? Hyperkinetic symptoms have been reported in a number of human patients who have suffered acute damage to the region of the globus pallidus. For example, buccofacial dyskinesia and truncal choreic movements have been seen after specific bilateral damage to the GP in humans following carbon monoxide poisoning or hemorrhage involving this region bilaterally $[48,69]$. Specific hyperkinetic movements include face and jaw movements that resemble tics, and involuntary finger movements during 'mental counting'. The walking gait of such a patient was described as choreic in nature [48]. These motor symptoms are accompanied by drastic changes in motivation and cognition $[48,69]$ which may be related to the motivational changes produced by our lesions [11]

\section{Conclusions}

In summary, this study mapped the location of two sites where neuron death produces two distinct sensory-triggered hyperkinetic syndromes. Damage to a site in the ventromedial globus pallidus (Fractions 207 and 208), exceeding a threshold of $60 \%$ bilateral neuron loss, produced the sensory-triggered treading syndrome but did not produce an increase in aversive taste reactions. The $\mathrm{T}$ syndrome site was approximately $0.8 \mathrm{~mm} \times 1.0 \mathrm{~mm} \times 1.0 \mathrm{~mm}$ in diameter. Damage to a ventromedial VP/SI site, exceeding $70 \%$ bilateral neuron loss, produced a combined syndrome of hyperkinetic treading and enhanced taste aversion to sensory triggers. The A + T syndrome site occupied the ventromedial corner of the ventral pallidum/substantia innominata (Fractions 215 and 216) and was ascertained to be approximately $1.0 \mathrm{~mm} \times 0.5 \mathrm{~mm} \times 1.0 \mathrm{~mm}$ in diameter (height, width, length). The determinant of which hyperkinetic syndrome was shown by an individual rat was found to be due to the placement of the lesion relative to these sites, rather than to the size or the suprathreshold severity of the excitotoxin lesions.

\section{Acknowledgements}

This work was supported by a National Institutes of Mental Health pre-doctoral fellowship MH-09838 (H.C.C.) and a National Institutes of Health grant NS-23959 (K.C.B.). We thank Roger Albin and Kevin Kaatz for their guidance in completing the immunohistochemistry. We thank Drs. Samuel Hicks, Connie D'Amato, and Roy Glover for help in developing the lesion identification procedure. We also thank the many undergraduates who helped complete the neuron 
counts, and Darlene Tansil for help with the behavioral scoring.

\section{References}

[1] Albin, R.L., Young, A.B. and Penney, J.B., Functional anatomy of the basal ganglia disorders, Trends Neurosci, 12 (1989) 366375.

[2] Albin, R.L., Reiner, A., Anderson, K.D., Penney, J.B. and Young, A.B., Striatal and nigral neuron subpopulations in rigid Huntington's disease: implications for the functional anatomy of chorea and rigidity-akinesia, Ann. Neurol., 27 (1990) 357-365.

[3] Albin, R.L., Young, A.B., Penney, J.B., Balfour, R., Anderson, K.D., Markel, D.S., Tourtellotte, W.W. and Reiner, A., Abnormalities of striatal projection neurons and $N$-methyl-D-aspartate receptors in pre-symptomatic Huntington's disease, New Engl. J. Med., 322 (1990) 1293-1298.

[4] Alexander, G.E., Delong, M.R. and Strick, P.L., Parallel organization of functionally segregated circuits linking basal ganglia and cortex, Ann. Rev. Neurosci., 9 (1986).

[5] Alheid, G.F. and Heimer, L., New Perspectives in basal forebrain organization of special relevance for neuropsychiatric disorders; the striatopallidal, amygdaloid and corticopetal components of substantia innominata, Neuroscience, 27 (1988) 1-39.

[6] Baldressarini, G.M. and Tarsy, D., Mechanisms underlying Tardive dyskinesia. In M.D. Yahrs (Ed.), The Basal Ganglia, Raven, New York, 1976, pp. 433-446.

[7] Bazzett, T.J., Becker, J.B., Kaatz, K.W. and Albin, R.L., Chronic intrastriatal dialytic administration of quinolinic acid produces selective neural degeneration, Exp. Neurol., 120 (1993) 177-185.

[8] Beal, M.F., Ferrante, R.F., Swartz, K.J. and Kowall, N.W., Chronic quinolinic acid lesions in rats closely resemble Huntington's disease, J. Neurosci., 11 (1991) 1649-1659.

[9] Berk, M.L. and Finkelstein, J.A., Efferent connections of the lateral hypothalamus area of the rat: an autoradiographic investigation, Brain Res. Bull., 8 (1982) 511-526.

[10] Berridge, K.C., Fentress, J.C. and Treit, D., A triggered hyperkinesia induced in rats by lesions of the corpus striatum, Exp. Neurol., 99 (1988) 259-268.

[11] Berridge, K.C. and Cromwell, H.C., Motivational-sensorimotor interaction controls aphagia and exaggerated treading after striatopallidal lesions, Behav. Neurosci., 104 (1990) 778-795.

[12] Boegman, R.K., Smith, Y. and Parent, A., Quinolinic acid does not spare striatal neuropeptide $\mathrm{Y}$-immunoreactive neurons, Brain Res., 415 (1987) 178-182.

[13] Bruyn, R.P. and Stoof, J.C., The quinolinic acid hypothesis in Huntington's chorea, J. Neurol. Sci., 95 (1990) 29-38.

[14] Bullen, J.G. and Helmsley, D.R., Sensory experience as a trigger in Gilles de la Tourette's syndrome, J. Behav. Ther. Exp. Psychiatry, 14 (1983) 197-201.

[15] Burns, A., Folstein, S., Brandt, J. and Folstein, M., Clinical assessment of irritability, aggression, and apathy in Huntington's and Alzheimer's disease, J. Nerv. Mental Dis., 178 (1990) 20-26.

[16] Carpenter, M.B. and Strominger, N.L., Corticostriate encephalitis and paraballism in the monkey. Evidence concerning distinctive functions of the globus pallidus, Arch. Neurol., 14 (1966) 241-253.

[17] Carpenter, M.B., Core Text in Neuroanatomy, Baltimore, Williams and Wilkins, 1978. 18 Omitted.

[19] Cromwell, H.C. and Berridge, K.C., Where does damage produce aversion: the ventral pallidum/substantia innominata or lateral hypothalamus? Brain Res., 624 (1993) 1-10.

[20] Crossman, A.R., Primate models of dyskinesia: the experimental approach to the study of basal ganglia-related involuntary movement disorders, Neuroscience, 21 (1987) 1-40.
[21] DeLong, M.R., Primate models of movement disorders of basal ganglia origin, Trends Neurosci., 13 (1990) 281-285.

[22] Denny-Brown, D. and Yanagisawa, I., The role of the basal ganglia in the initiation of movement. In M.D. Yahrs, The Basal Ganglia, Raven, New York, 1976, pp. 115-150.

[23] Difiglia, M., Excitotoxic injury of the neostriatum: a model for Huntington's disease, Trends Neurosci, 13 (1990) 286-289.

[24] Fuller, T.A., Russhen, F.T. and Price, J.T., Sources of presumptive glutamatergic/aspartatergic afferents to the rat ventral striatopallidal region, J. Comp. Neurol., 258 (1987) 317-338.

[25] Gilles de la Tourette, G., Etude sur une affection nerveuse caractarisee de l'incoordination motrice accompagnee d'echolalie et de copralalie, Arch. Neurol., 9 (1885) 19-42+158-200.

[26] Grill, H.J. and Norgren, R., Neurological tests an behavioral deficits in chronic thalamic and chronic decerebrate rats, Brain Res., 143 (1978) 299-312.

[27] Grill, H.J. and Berridge, K.C., Taste reactivity as a measure of the neural control of palatability. In J.M. Sprague and A.N. Epsteins (Eds.), Progress in Psychobiology and Physiological Psychology, Vol. 2, Orlando, FL, 1985, pp. 1-62.

[28] Groenewegen, H.J., Berendse, H.W., Wolters, J.G. and Lohman, A.H., The anatomical relationship of the prefrontal cortex with the striatopallidal system, the thalamus and the amygdala: evidence for a parallel organization, Prog. Brain Res., 85 (1990) 95-116; discussion 116-118.

[29] Groenewegen, H.J. and Berendse, H.W., Connections of the subthalamic nucleus with ventral striatopallidal parts of the basal ganglia in the rat, J. Comp. Neurol., 294 (1990) 607-622.

[30] Groenewegen, H.J., Berendse, H.W., Meredith, G.E., Haber, S.N., Voorn, P., Wolters, J.G. and Lohman, A.H., Functional anatomy of the ventral limbic system-innervated striatum. In P. Willner and J. Scheel-Krugers (Eds.), The Mesolimbic Dopamine System, Wiley and Sons, New York, 1991, pp. 19-60.

[31] Grove, E.A., Efferent connections of the substantia innominata in the rat, J. Comp. Neurol., 277 (1988) 347-364.

[32] Grove, E.A., Neural associations of the substantia innominata in the rat: afferent connections, J. Comp. Neurol., 277 (1988) $315-346$.

[33] Gundersen, H.J., Bagger, P., Bendtsen, T.F., Evans, S.M., Korbo, L., Marcussen, N., Moller, A., Nielsen, K., Nyengaard, J.R. and Pakkenberg, B., The new stereological tools: dissector, fractionator, nucleator, and point sampled intercepts and their use in pathological research and diagnosis, APMIS, 96 (1988) $857-881$.

[34] Haber, S.N. and Nauta, W.J., Ramifications of the globus pallidus in the rat as indicated by patterns of immunohistochemistry, Neuroscience, 9 (1983) 245-260.

[35] Haber, S.N. and Watson, S.J., The comparative distribution of enkephalin, dynorphin and substance $P$ in the human globus pallidus and basal forebrain, Neuroscience, 14 (1985) 1011-1024.

[36] Haber, S.N., Groenewegen, H.J., Grove, E.A. and Nauta, W.J., Efferent connections of the ventral pallidum: evidence of a dual striato pallidofugal pathway, J. Comp. Neurol., 235 (1985) 322335.

[37] Hantraye, P., Riche, D., Maziere, M. and Isacson, O., A primate model of Huntington's disease:behavioral and anatomical studies of unilateral excitotoxic lesions of the caudate-putamen in the baboon, Exp. Neurol., 108 (1990) 91-104.

[38] Hazrati, L.N. and Parent, A., The striatopallidal projection displays a high degree of anatomical specificity in the primate, Brain Res., 592 (1992) 213-27.

[39] Heimer, L. and Wilson, R.D., The subcortical projections of the allocortex: similarities in the neural associations of the hippocampus, the pyriform cortex, and the neocortex. In M. Santinis (Ed.), Golgi Centennial Symposium, Raven, New York, 1975, pp. $177-193$.

[40] Heimer, L., Zaborsky, L., Zahm, D.S. and Alheid, G.F., The 
ventral striatopallidothalamic projection: I. The striato-pallidal link originating in the striatal parts of the olfactory tubercle, $J$. Comp. Neurol., 255 (1987) 571-591.

[41] Heimer, L., Zahm, D.S., Churchill, L., Kalivas, P.W. and Wohltmann, C., Specificity in the projection patterns of the acumbel core and shell in the rat, Neuroscience, 41 (1991) 89-125.

[42] Jackson, A. and Crossman, A.R., Experimental choreoathetosis produced by injection of a gamma-aminobutyric acid antagonist into the lentiform nucleus in the monkey, Neurosci. Lett., 46 (1984) 41-45.

[43] Jones, D.L. and Mogenson, G.J., Nucleus accumbens to globus pallidus GABA projection: electrophysiological and iontophoretic investigations, Brain Res., 188 (1980) 93-105.

[44] Kanazawa, I., Kimura, M., Murata, M., Tanaka, Y. and Cho, F., Choreic movements in the macaque monkey induced by kainic acid lesions of the striatum combined with L-dopa. Pharmacological, biochemical and physiological studies on neural mechanisms, Brain, 113 (1990) 509-535.

[45] Korenyi, C., Whittier, J.R. and Conchado, D., Stress in Huntington's disease, Dis. Nerv. Syst., 33 (1972) 339-344.

[46] Kurlan, R., Lichter, D. and Hewitt, D., Sensory tics in Tourette's syndrome, Neurology, 39 (1989) 731-734.

[47] Lange, H., Thorner, G., Hopf, A. and Schroder, K.F., Morphometric studies of the neuropathological changes in choreatic diseases, J. Neurol. Sci., 28 (1976) 401-425.

[48] LaPlane, D., Baulac, M., Widlocher, D. and Dubois, B., Pure psychic akinesia with bilateral lesions of the basal ganglia, $J$. Neurol. Neurosurg. Psychiatry, 47 (1984) 377-385.

[49] Lasiter, P.S. and Garcia, J., A methoxyflurane delivery system for stereotaxic surgery, Brain Res. Bull., 13 (1984) 457-460.

[50] Levine, M.S. and Schwartzbaum, J.S., Sensorimotor functions for the striatopallidal system and lateral hypothalamus and consummatory behavior in rats, J. Comp. Physiol. Psychol., 85 (1973) 615-635

[51] McGeorge, A.J. and Faull, R.L., The organization of the projection from the cerebral cortex to the striatum in the rat, Neuroscience, 29 (1989) 503-537.

[52] Mogenson, G.J. and Nielsen, M.A., Evidence that an accumbens to subpallidal GABAergic projection contributes to locomotor activity, Brain Res. Bull., 11 (1983) 309-314.

[53] Mogenson, G.J., Swanson, L.W. and Wu, M., Neural projections from nucleus accumbens to globus pallidus, substantia innominata, and lateral preoptic-lateral hypothalamic area: an anatomical and electrophysiological investigation in the rat, $J$. Neurosci., 3 (1983) 189-202.

[54] Mogenson, G.J., Swanson, L.W. and Wu, M., Evidence that projections from substantia innominata to zona incerta and mesencephalic locomotor region contribute to locomotor activity, Brain Res., 334 (1985) 65-76.

[55] Mogenson, G.J., Ciriello, J., Garland, J. and Wu, M., Ventral pallidum projections to mediodorsal nucleus of the thalamus: an anatomical and electrophysiological investigation in the rat, Brain Res., 404 (1987) 221-230.

[56] Mogenson, G.J., Wu, M. and Tsai, C.T., Subpallidal-pedunculopontine projections but not subpallidal-mediodorsal thalamus projections contribute to spontaneous exploratory locomotor activity, Brain Res., 485 (1989) 396-8.

[57] Narabayshi, H., Chida, T. and Kondo, T., Analysis of two factors in influencing involuntary movement: psychological stress and motor effect. In L.J. Poirer, T.L. Sourkes and P. Bedards (Eds.), Advances in Neurology, New York, 1979, pp. 361-371.

[58] Nauta, W.J. and Mehler, W.R., Projections of the lentiform nucleus in the monkey, Brain Res., 1 (1966) 3-42.

[59] Nauta, H.J.W., Projection of the pallidal complex: an autoradiographic study in the cat, Neuroscience, 4 (1979) 1853-1873.
[60] Nauta, H.W.J., A proposed conceptual reorganization of the telencephalon, Neuroscience, 4 (1979) 1875-1881.

[61] Nauta, W.J. and Domesick, V.B., Afferent and efferent relationships of the basal ganglia, Ciba Found. Symp., 107 (1984) 3-29.

[62] Nauta, W.J.H., Reciprocal links of the corpus striatum with the cerebral cortex and limbic system: a common substrate for movement and thought? In W. Muellers (Ed.), Neurology and Psychiatry: a Common Substrate for Movement and Thought?, Karger, Basel, 1989, pp. 43-63.

[63] Paxinos, G. and Watson, C., The Rat Brain in Stereotaxic Coordinates, Academic Press, New York, 1982.

[64] Penney Jr., J. and Young, A.B., Striatal inhomogeneities and basal ganglia function, Mov. Disord., 1 (1986) 3-15.

[65] Reiner, A., Albin, R.L., Anderson, K.D., D’Amato, C.J., Penney, J.B. and Young, A.B., Differential loss of striatal projection neurons in Huntington disease, Proc. Natl. Acad. Sci. USA, 85 (1988) 5733-5737.

[66] Sanberg, P.R., Calderon, S.F., Giordiano, M., Tew, J.M. and Norman, A.B., The quinolinic acid model of Huntington's disease: locomotor abnormalities, Exp. Neurol., 105 (1989) 45-53.

[67] Schneider, J.S., Role of the basal ganglia in a chemically induced dyskinesia in rat, Exp. Neurol., 84 (1984) 524-532.

[68] Singer, H.S., Hahn, I.H. and Moran, T.H., Abnormal dopamine uptake sites in postmortem striatum from patients with Tourette's syndrome, Ann. Neurol., 30 (1991) 558-562.

[69] Starkstein, S.E., Berthier, M.L. and Leguardia, R., Psychic akinesia following bilateral pallidal lesions, Intl. J. Psychiat. Med., 19 (1989) 155-164.

[70] Tarsy, D., Pycock, C.J., Meldrum, B.S. and Marsden, C.D., Focal contralateral myoclonus produced by inhibition of GABA action in the caudate nucleus of rats, Brain, 101 (1978) 143-162.

[71] Thompson, P.D., Berardelli, A., Rothwell, J.C., Day, B.L., Dick, P., Benecke, R. and Marsden, C.D., Pathophysiology of tics and chorea. In R. Benecke, B. Conrad and C.D. Marsdens (Eds.), Motor Disturbances I, academic Press, London, 1987.

[72] Whetsell, W.O. and Schwarcz, R., Prolonged exposure to submicromolar concentrations of quinolinic acid causes excitotoxic damage in organotypic cultures of rat corticostriatal system, Neurosci. Lett., 97 (1989) 271-275.

[73] Wilson, S.A.K., An experimental research into the anatomy and physiology of the corpus striatum, Brain, 36 (1914) 427-492.

[74] Winn, P., Excitotoxins as tools for producing brain lesions. In P.M. Conns (Ed.), Lesions and Transplantations, Academic Press, New York, 1991, pp. 16-27.

[75] Young, A.B., Albin, R.L. and Penney, J.B., Neuropharmacology of basal ganglia functions: relationship to pathophysiology of movement disorders. In A.R. Crossman and M.A. Sambrooks (Eds.), Neural Mechanisms of Movement Disorders, J. Libbey Press, London, 1989, pp. 17-28.

[76] Zaborsky, L., Eckenstein, F., Leranth, C., Oertel, W., Schemechel, D., Alones, V. and Heimer, L., Cholinergic cells of the ventral pallidum: a combined electron microscopic immunocytochemical, degeneration and HRP study, Soc. Neurosci. Abstr., 10 (1984) 8.

[77] Zahm, D.S., The ventral striatopallidal parts of the basal ganglia in the rat-II. Compartmentation of ventral pallidal efferents, Neuroscience, 30 (1989) 33-50.

[78] Zahm, D.S. and Heimer, L., Two transpallidal pathways originating in the rat nucleus accumbens, J. Comp. Neurol., 302 (1990) 437-446.

[79] Zahm, D.S. and Brog, J.S., On the significance of subterritories in the 'accumbens' part of the rat ventral striatum, Neuroscience, 50 (1992) 751-67. 